\title{
Cardiac and skeletal muscle disorders caused by mutations in the intracellular $\mathrm{Ca}^{2+}$ release channels
}

\author{
Silvia G. Priori1,2 and Carlo Napolitano'1 \\ ${ }^{1}$ Molecular Cardiology, Istituto di Ricovero e Cura a Carattere Scientifico Fondazione Maugeri, Pavia, Italy. \\ 2Department of Cardiology, University of Pavia, Pavia, Italy.
}

\begin{abstract}
Here we review the current knowledge about the mutations of the gene encoding the cardiac ryanodine receptor $(R y R 2)$ that cause cardiac arrhythmias. Similarities between the mutations identified in the RyR2 gene and those found in the gene $R y R 1$ that cause malignant hyperthermia and central core disease are discussed. In vitro functional characterization of $R y R 1$ and $R y R 2$ mutants is reviewed, with a focus on the contribution that in vitro expression studies have made to our understanding of related human diseases.
\end{abstract}

\section{Introduction}

Rapid mobilization of calcium from the sarcoplasmic reticulum (SR) into the cytosol triggers activation of contractile elements, and it is therefore a fundamental process in the physiology of heart and muscles. The channels that regulate the duration and amplitude of calcium efflux from the SR are the ryanodine receptors (RyRs). Three subtypes of these proteins exist: RyR 1 is mainly expressed in skeletal muscle, RyR2 is highly represented in cardiac tissue, and RyR3 is preferentially expressed in the brain. In this review we will focus on the genetic diseases that affect structure and function of the cardiac isoform of the ryanodine receptor, RyR2, causing development of ventricular arrhythmias and sudden cardiac death. Since genetic disorders affecting the skeletal muscle isoform (1) of the ryanodine receptor, RyR1, were described 10 years before those affecting RyR2 (2), and since observations initially made about mutations in the RyR1 gene can be extended to those identified in the $R y R 2$ gene, we will discuss selected aspects of RyR1 diseases in light of their similarities with RyR2 diseases.

\section{The cardiac ryanodine receptor and intracellular calcium release}

\section{Structure of the cardiac ryanodine receptor, $R y R 2$}

The cardiac ryanodine receptor is a protein composed of 4,967 amino acids with a molecular weight of $565 \mathrm{kDa}$ (3) that is encoded by the large $R y R 2$ gene located on chromosome 1 (1q42-q43) (4). This is a large gene spanning $790 \mathrm{~kb}$ of genomic DNA with 105 exons (5). The functional channel is a tetramer that derives from the coassembly of 4 of the subunits encoded by the RyR2 gene, resulting in a 2.2-megadalton complex (Figure 1). Each RyR2 monomer is composed of 2 major structural regions. The carboxy terminus (spanning approximately from aa 4,500 to the end of the protein) is the pore-forming region, which is composed of an even, but yet undefined, number of

Nonstandard abbreviations used: ARVD2, arrhythmogenic right ventricular cardiomyopathy type 2; CCD, central core disease; CPVT, catecholaminergic polymorphic ventricular tachycardia; DAD, delayed afterdepolarization; $\mathrm{MH}$, malignant hyperthermia; PKA, protein kinase A; RyR, ryanodine receptor; SR, sarcoplasmic reticulum; VT, ventricular tachycardia.

Conflict of interest: The authors have declared that no conflict of interest exists.

Citation for this article: J. Clin. Invest. 115:2033-2038 (2005).

doi:10.1172/JCI25664. transmembrane segments (4-10 for each monomer, most likely 6) $(6,7)$. This portion of the protein, despite being relatively small, is the most important, because it contains sequences that control RyR2 localization and oligomerization (8) and is sufficient to form a functional $\mathrm{Ca}^{2+}$ release channel. The second structural region, which includes nine-tenths of the protein, corresponds to the large cytoplasmic domain that performs regulatory functions.

\section{Function of the cardiac ryanodine receptor, RyR2}

During the plateau phase of the action potential, the opening of L-type voltage-dependent calcium channels (dihydropyridine receptors) creates a small influx of calcium ions that triggers a massive release of calcium from the SR by opening the RyR2 channels: this process is called calcium-induced calcium release and is the pivotal mechanism that controls coordination between electrical and mechanical functions in cardiac myocytes (9). In the ventricles of mammals, the ryanodine receptors are located in the proximity of the surface membrane and of the T tubules; this location is strategic to ensure a prompt interaction of RyR2 with L-type calcium channels (10). Thanks to this architecture, for every L-type calcium channel that opens, 4-6 ryanodine receptors are synchronously activated to generate a $\mathrm{Ca}^{2+}$ spark (11). A cluster of approximately 100 ryanodine receptor channels and 25 L-type calcium channels creates a functional unit called a junction, or couplon (12). There are approximately 10,000 couplons in a ventricular myocyte that are simultaneously activated by $\mathrm{Ca}^{2+}$ influx for every heartbeat (13). The coordination in opening and closing (14) is critical for timing of intracellular calcium release to the systolic phase of the cardiac cycle, which thus ensures functional silence during diastole.

\section{RyR2 and its macromolecular complex}

The ryanodine receptor interacts with a multitude of accessory proteins to form a macromolecular calcium release complex. Junctin, triadin, and calsequestrin are the most important proteins that interact with RyR2 at the intra-SR level. It has been proposed that other proteins located in the SR, such as sarcalumenin and calreticulin, may also interact with RyR 2 and be part of its macromolecular complex. Most of the regulatory functions are accomplished by proteins binding to the cytosolic portion of RyR2, such as calmodulin, protein phosphatase 1 , protein phosphatase $2 \mathrm{~A}$, 


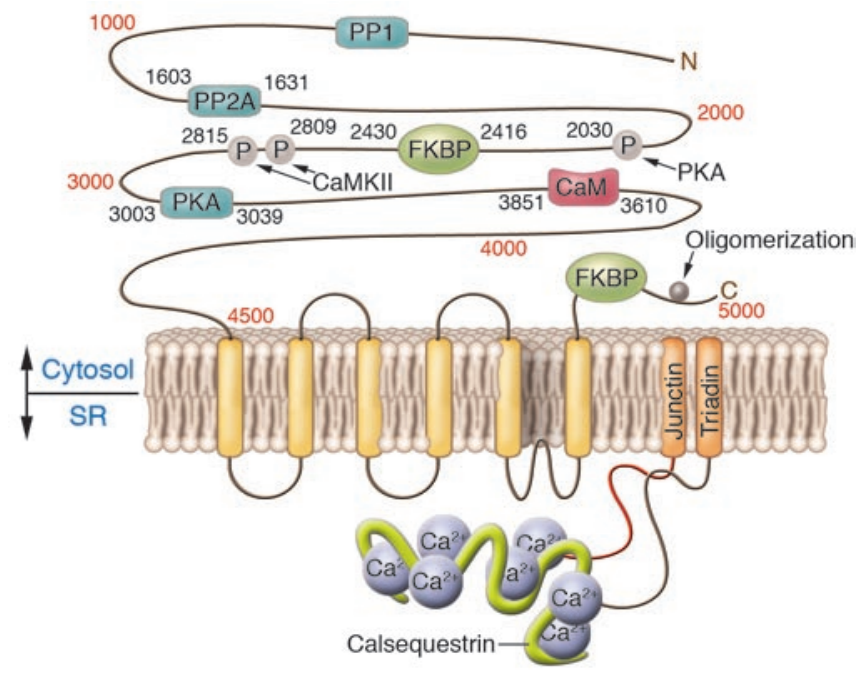

Figure 1

Schematic showing the predicted structure of the cardiac ryanodine receptor, RyR2, including the sites of interaction with ancillary proteins and the phosphorylation sites. Calsequestrin, junctin, and triadin, proteins interacting with ryanodine receptor in the $S R$, are also depicted. PP, protein phosphatase; $\mathrm{P}$, phosphorylation sites; $\mathrm{CaM}$, calmodulin; CaMKII, calmodulin-dependent protein kinase II. Adapted with permission from the Journal of Molecular and Cellular Cardiology (13).

protein kinase A (PKA), calmodulin-dependent protein kinase II, calcineurin, and PKC isoforms $\alpha$ and $\beta$ (15). Among the cytosolic ligands, the peptidyl-prolyl isomerase FKBP12.6 (calstabin 2) has been extensively studied. It is a $12.6-\mathrm{kDa}$ protein that binds to a specific region of RyR2 that, according to some authors, encompasses aa 2,416-2,430; it should be noted, however, that this binding site has been disputed $(16,17)$. FKBP12.6 stabilizes the closed state of the RyR2 channel, thus preventing aberrant calcium leakage $(18,19)$. The binding of FKBP12.6 to RyR2 is thought to be modulated by PKA-mediated phosphorylation that decreases the binding affinity of RyR2 for FKBP12.6, thus increasing the open probability of the channel and amplifying its response to calcium-dependent activation $(18,19)$. However, the role of this molecule in PKA phosphorylation has also been questioned, at least with regard to serine 2,030 (a major RyR2 phosphorylation site) (Figure 1) (20).

\section{Ryanodine receptors and human diseases}

\section{Mutations in RyR1, central core disease, and malignant byperthermia}

The first evidence that human genetic diseases may be associated with mutations of ryanodine receptors came from studies performed on the skeletal muscle isoform, RyR1. Predisposition to 2 diseases, malignant hyperthermia $(\mathrm{MH})$ and central core disease (CCD), was associated with RyR1 mutations $(21,22)$. MH is characterized by acute hyperpyrexia that develops during or immediately after general anaesthesia. The disease shows paroxysmal manifestations that occur in carriers of a mutation upon exposure to volatile anaesthetics; in this setting, therefore, the mutations create a substrate that becomes apparent only in the presence of the specific triggering agent: i.e., it is needed, but not sufficient, to cause the phenotype. CCD is a rare neuromuscular disorder that causes weakness in the muscles of the legs and, less frequently, in other muscles. Its name derives from the presence of abnormal "cores" identified at histological examination in type I muscle fibers that also present sarcomeric disorganization, lack of mitochondria, and lack of oxidative activity.

Molecular genetics and functional characterization of RyR1 mutants. Most of the mutations identified in RyR 1 cluster in 3 regions: the amino terminus (including approximately the region between aa 35 and aa 614), a central region (spanning aa 1,787-2,458), and the carboxy terminus (extending from aa 4,796 to aa 4,973 ). It should be noted, however, that as more families with RyR1 mutations are being identified, the boundaries of these regions are becoming less well defined. It is interesting to observe that in the last 3 years, 9 distinct mutations have been reported in the region between aa 3,527 and aa 4,651 - a portion of the gene that was until then devoid of mutations. In analogy with what has happened with most genetic diseases, the spreading of genetic analysis in families with $\mathrm{CCD}$ and $\mathrm{MH}$ is broadening the spectrum of the clinical phenotypes and is disclosing unexpected findings. For example, despite that both CCD and $\mathrm{MH}$ are inherited as autosomal dominant conditions, CCD families with homozygous mutations have been reported (23), and 1 family with compound heterozygous mutations has been identified presenting with an overlapping phenotype of susceptibility to $\mathrm{MH}$ combined with a mild form of CCD (24).

In a few instances, $R y R 1$ mutations have been identified in patients with phenotypes unrelated to $\mathrm{MH}$ and CCD: for example, a proline-to-serine substitution at position 3,527 (25) and a splice error (26) have been associated with multi-minicore disease, a congenital myopathy transmitted as a recessive trait that manifests with hypotonia and generalized weakness in axial and proximal limb muscles. It seems premature to decide, based on these sporadic reports, whether the latter disease is allelic to CCD and $\mathrm{MH}$; it seems more appropriate to wait until more genotyping data become available. A similar conservative approach is recommended for discussion of RyR2 mutations identified in a few families diagnosed with an atypical form of right ventricular cardiomyopathy, arrhythmogenic right ventricular cardiomyopathy type 2 (ARVD2) (see below).

Functional characterization of mutant proteins is a very important step in the understanding of the pathophysiological mechanisms of a genetic disease. In the next few paragraphs, we will try to summarize the key results of the in vitro studies of RyR1 mutants and will highlight data that clarify how mutations in the same gene may cause 2 apparently unrelated diseases such as CCD and MH.

Most of the RyR1 mutations characterized in different in vitro systems lead to a gain of function. Richter et al. (27) showed that the G2434R mutation identified in patients with $\mathrm{MH}$ enhances the sensitivity of RyR1 to $\mathrm{Ca}^{2+}$ and caffeine. Similar findings were reported by Yang et al. (28) when they expressed 6 common RyR1 mutants found in MH patients (R163C, G341R, R614C, R2163C, V2168M, and R2458H) in myotubes differentiated from cells lacking endogenous $R y R 1$, and by Wehner et al. (29) when they characterized RyR 1 mutants in primary human myotubes cultured from carriers of RyR1 mutations. Overall, these studies suggest that the leading pathophysiological substrate for MH is RyR1, which, although it functions normally at resting conditions, presents enhanced sensitivity to activators and 


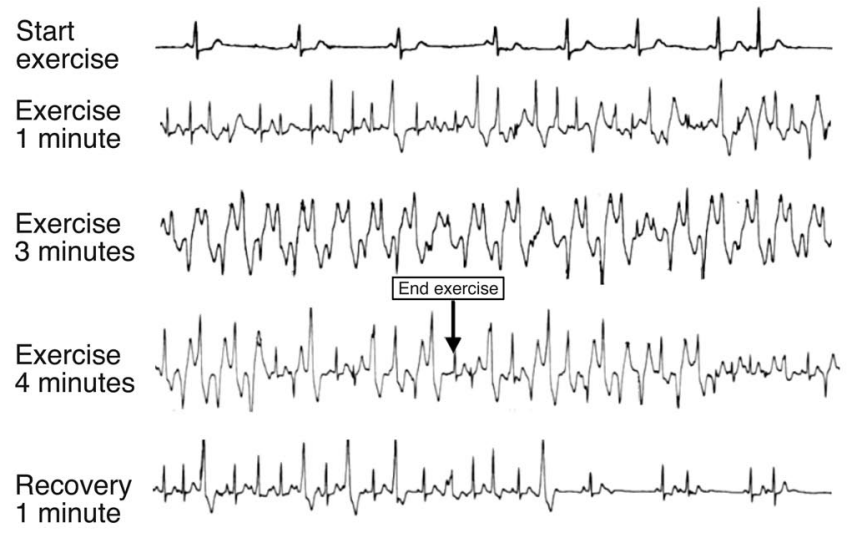

Figure 2

Exercise stress test in a patient with polymorphic VT and RyR2 mutation. Ventricular arrhythmias are observed with a progressive worsening during exercise. Typical bidirectional VT develops after 3 minutes of exercise with a sinus heart rate of approximately $120 \mathrm{bpm}$. Arrhythmias rapidly recede during recovery.

reduced sensitivity to endogenous inhibitors $\left(\mathrm{Ca}^{2+}\right.$ and $\left.\mathrm{Mg}^{2+}\right)$. Abnormal response to activators may therefore precipitate the clinical manifestations.

Different properties of RyR1 mutants have been highlighted in the study of defects identified in CCD patients, and at least 3 other abnormalities have been reported: (a) mutations occurring in exons 101 and 102 were shown to make RyR1 leaky in basal conditions without altering the response to pharmacological modulators with reduced intracellular calcium stores (30); (b) uncoupling of sarcolemmal excitation (voltage-gated $\mathrm{Ca}^{2+}$ channel) from SR $\mathrm{Ca}^{2+}$ release was reported for some CCD mutations $(31,32)$; and (c) mutations located in the amino terminus of the RyR1 protein have been reported to share the common characteristic of increasing $\mathrm{Ca}^{2+}$ release (leak) at negative (resting) membrane potentials (32).

In summary, some hypotheses have been advanced to explain the different phenotypes associated with RyR1 mutations. DNA defects found in $\mathrm{MH}$ patients appear to produce channels that have a normal behavior at rest but overreact to exogenous activators. On the contrary, the majority of CCD mutants produce a channel that is leaky at base line, depletes the SR, and may also cause electrical uncoupling between RyR1 and the voltage-gated $\mathrm{Ca}^{2+}$ channels. To add to this interpretation, Dirksen and Avila (33) suggested that, since the degree of $\mathrm{Ca}^{2+}$ leakage and EC uncoupling induced by different mutations is variable, the combination of these effects may represent a critical modulator of the clinical phenotype.

\section{Mutations in RyR2 and catecholaminergic polymorphic ventricular tachycardia}

Mutations in the RyR2 gene have been identified in families and in sporadic patients affected by catecholaminergic polymorphic ventricular tachycardia (CPVT). This disease was initially described in 1978 (34) and more extensively characterized in 1995 (35). The name derives from the clinical evidence that these patients develop a typical polymorphic or bidirectional ventricular tachycardia (VT) that leads to syncopal episodes during catecholamine release in the setting of emotion or exercise (Figure 2). In most instances, CPVT is inherited as an autosomal dominant disease, and approximately $30 \%$ of patients have a family history of juvenile sudden death and/or stress-related syncope (35). The first clinical manifestations of the disease appear during childhood, although late-onset forms have been described (36).

Linkage studies localized the CPVT gene on the long arm of chromosome 1, 1q42-q43 (37). Thus the human cardiac ryanodine receptor gene (RyR2) that mapped in that region became a plausible candidate gene for CPVT. We performed screening of the coding region of this gene in DNA obtained from CPVT patients and were able to identify pathogenetic mutations (2). An uncommon autosomal recessive variant of CPVT has been identified and linked to chromosome 1p13-p21 (38); shortly after the publication of linkage data, mutations were found in the gene Casq2 that encodes for calsequestrin, thus confirming the view that CPVT is a disease caused by intracellular calcium handling abnormalities $(39,40)$.

Treatment ofCPVTpatients. Antiadrenergic treatment with $\beta$-blockers is the mainstay of therapy for CPVT patients, and it is indicated in all patients with clinical diagnosis $(35,36)$. No data are available to define whether asymptomatic carriers of RyR2 mutations should also be treated with prophylactic $\beta$-blockers. These drugs reduce the number of runs of polymorphic VT and their rate and duration in the majority of patients, but they usually fail to completely suppress ventricular tachyarrhythmias. In those patients in which $\beta$-blockers do not prevent the recurrence of sustained VT, it is recommended to consider the implantation of a cardioverter defibrillator (41).

Interestingly, a single group of investigators claimed the identification of RyR2 mutations in families diagnosed with an atypical form of right ventricular cardiomyopathy, ARVD2 (5), that combines modest structural abnormalities of the right ventricle with typical stress-induced polymorphic VTs. The presence of RyR2 mutations in patients with the clinical diagnosis right ventricular cardiomyopathy has not been confirmed by other groups, and, at the present time, whether mutations in RyR2 cause ARVD2 remains a matter of debate that awaits further data.

Role of genetic testing in CPVT. Approximately $40 \%$ of CPVT patients carry a mutation on the RyR2 gene; as a consequence, it is recommended that all patients with this clinical diagnosis undergo genetic analysis. Because DNA analysis can be extended to all family members to identify those who are asymptomatic but genetically affected, it is important to know whether the clinically affected proband carries the mutation. Silent-mutation carriers should receive prophylactic treatment with $\beta$-blockers with the objective of preventing sudden cardiac death $(35,36)$. At the present time, no genotype-phenotype correlations have been established

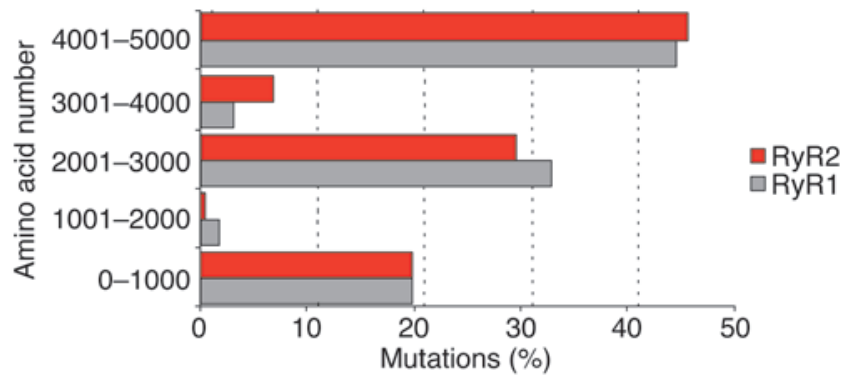

\section{Figure 3}

Bar graph showing the similar clustering of mutations in RyR1 (including both MH and CCD phenotypes) and RyR2 (both CPVT and ARVD2). 


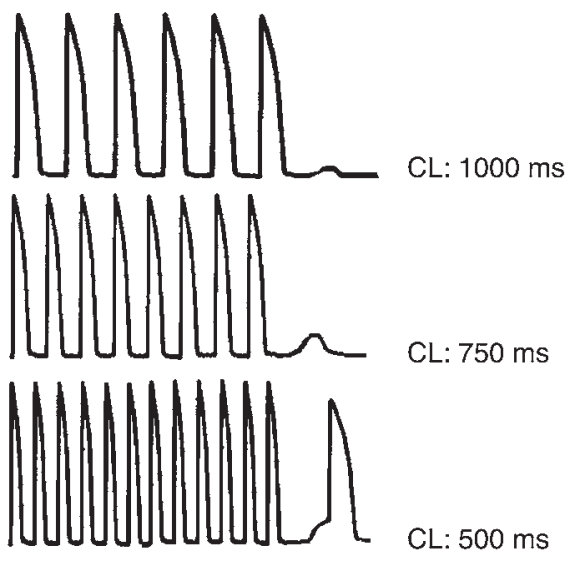

Figure 4

Representation of a cardiac action potential, showing DADs. DAD amplitude increases and DAD coupling interval decreases upon cycle length $(C L)$ reduction. With further increase in frequency of stimulation, an automatic beat is generated from the DAD.

in CPVT, and it will be important to collect data on larger numbers of genotyped individuals with a long clinical follow-up to determine whether it is possible to define risk of cardiac events or response to therapy based on the genetic defect $(42,43)$.

Molecular genetics and functional characterization of RyR2 mutants. All RyR2 mutations identified so far in CPVT patients are singlebase-pair substitutions that lead to the replacement of a highly conserved amino acid. This is at variance with RyR1 mutations found in $\mathrm{MH}$ and CCD patients, where splice errors and deletions have also been reported. The distribution of mutations on the predicted topology of the proteins, however, is identical for RyR1 and RyR2. Most RyR2 mutations are clustered in the amino terminus, in the FKBP12.6-binding domains, and in the transmembrane domains of the protein (Figure 3). Very few RyR2 mutations have been functionally characterized, so it is still difficult to define whether functional differences exist among mutations and whether they account for the severity of the phenotype (33). Furthermore, some of the first mutations identified have been expressed by different groups yielding different results.

The first $R y R 2$ mutation to be functionally characterized was the mouse homolog of the mutation (R4497C) identified in the large Italian family in which the discovery that RyR2 is the CPVT gene was made (2). This mutation is a mutational "hot spot" that has now been identi-

B

Figure 5 fied in additional families and in 1 sporadic individual $(44,45)$. In vitro studies have proven that the R4497C mutant channel shows important behavioral abnormalities as compared with the wildtype channel (46): R4497C-RyR2 presents enhanced resting activity, increased single-channel open probability, and accentuated sensitivity to caffeine activation leading to $\mathrm{Ca}^{2+}$ overload.

When other RyR2 mutations were expressed in stable human embryonic kidney cell lines (HEK 293), it became clear that RyR2 mutations that cause CPVT share some common features: they increase sensitivity to luminal calcium activation and enhance the propensity for spontaneous calcium release from the SR. These properties would facilitate the development of delayed afterdepolarizations (DADs) and triggered arrhythmias (see below) and therefore establish a link between the molecular defect and the susceptibility to adrenergically mediated arrhythmias (47).

A novel and still debated interpretation of the consequence of $R y R 2$ mutation was provided by Wehrens et al. (48), who suggested that the key feature of CPVT mutants is a reduced affinity of the channel for the binding of the regulatory protein FKBP12.6, leading to a channel that releases calcium during diastole. George et al. (49) did not confirm the data of Wehrens et al.: they found that mutant channels had a normal RyR2/FKBP12.6 interaction but an abnormally augmented response to isoproterenol and forskolin, resulting in excessive calcium release. The same group recently reported another interesting observation (50) when they expressed mutations identified in ARVD2 patients (5). Interestingly, 1 of the 4 mutants studied (50) was not associated with the conventional gain of function (increased response to RyR2 agonists that promote calcium release from the SR, such as caffeine) but rather induced a loss of function. In a speculative editorial, Gomez and Richard (51) put forward a highly provocative hypothesis by suggesting that a loss-of-function mutation would reduce $\mathrm{Ca}^{2+}$ release
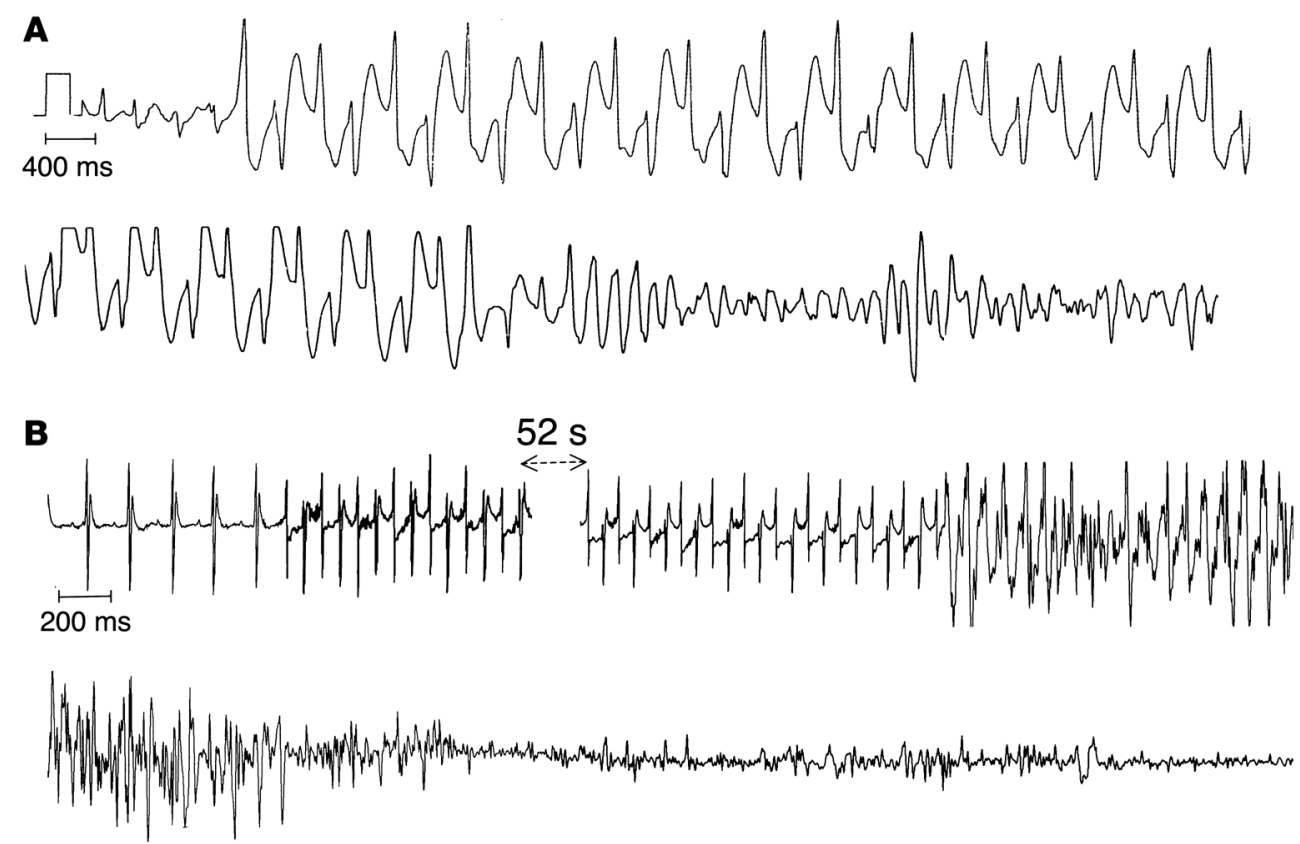

Bidirectional VT and ventricular fibrillation in CPVT. (A) ECG recording of bidirectional VT degenerating into ventricular fibrillation in a CPVT patient. (B) ECG recording of a bidirectional VT degenerating into ventricular fibrillation in a RyR2+/RyRR4496C mouse. 
from RyR2 and that this could in turn slow the inactivation of the current conducted by the sarcolemmal calcium-dependent voltage channel and prolong cardiac action potential. In this view, arrhythmias triggered by loss-of-function mutations in RyR2 could still be triggered, but by early afterdepolarizations (EADs) rather than by DADs. Albeit appealing, this hypothesis is in contrast with common knowledge that EAD-induced arrhythmias occur in the setting of bradycardia; EADs therefore could hardly account for adrenergically mediated arrhythmias that originate during sinus tachycardia. A further improvement in the understanding of the pathophysiology of RyR2-related arrhythmias should now be provided by knock-in animal models.

Overall, functional characterization of mutants performed so far seems to agree on the presence of an increased calcium release response upon adrenergic stimulation and on a lower threshold for calcium spilling from the SR. All these dysfunctions are likely to promote the development of DADs and triggered arrhythmias. Less agreement exists on the mechanisms that mediate these abnormal responses, and, specifically, the role of FKBP12.6 needs to be further elucidated.

From functional characterization of mutants to electrophysiological mechanisms of CPVT. Even before the identification of the gene responsible for CPVT, the hypothesis that arrhythmias were initiated by DADs had been advanced based on the observation that the bidirectional VT observed in CPVT patients closely resembles digitalis-induced arrhythmias. A lot is known about the electrophysiological mechanisms underlying digitalis-induced arrhythmias: sound experimental data have demonstrated that in the presence of digitalis-induced intracellular calcium overload the $\mathrm{Na}^{+} / \mathrm{Ca}^{2+}$ exchanger gets activated in the attempt to reduce calcium and exchanges $1 \mathrm{Ca}^{2+}$ for $3 \mathrm{Na}^{+}$, thus generating a net inward current (the so-called transient inward current). This depolarizing current promotes diastolic depolarizations (called delayed afterdepolarizations, or DADs) (52) that may reach the threshold for $\mathrm{I}_{\mathrm{Na}}$ activation and elicit premature action potentials. This mechanism for arrhythmia initiation is called triggered activity. Several in vitro studies have demonstrated that $\beta$-adrenergic stimulation induces DADs both in Purkinje fibers and in cardiac myocytes $(52,53)$. Based on the above-mentioned information, it becomes appeal- ing to speculate that RyR2 mutations identified in CPVT patients promote the development of calcium overload in concomitance with sympathetic stimulation and the activation of the $\mathrm{Na}^{+} / \mathrm{Ca}^{2+}$ exchanger, thus leading to the development of DADs and triggered arrhythmias (Figure 4). This hypothesis still awaits conclusive experimental support: the availability of the recently described knock-in mouse carrier of the R4496C RyR2 mutation (54), in which bidirectional VTs can be induced (Figure 5), will allow demonstration of whether RyR2 mutations lead to the development of DADs as the initiating mechanism for cardiac arrhythmias.

\section{Conclusions}

In the last few years, advancements in understanding the uncommon inherited arrhythmogenic disorder CPVT have given momentum to the study of the regulation of intracellular calcium handling.

The discovery of DNA mutations that modify the intracellular calcium release channel ryanodine receptor has highlighted the pivotal role of RyR2 in the control of intracellular calcium and has brought to general appreciation the link between RyR2 and electrophysiological properties of the cardiac myocytes. These observations are of major importance because they may lead to innovative curative approaches that may apply not only to patients with CPVT, but also to individuals with acquired arrhythmias associated with dysfunctional intracellular calcium handling, such as heart failure patients. As it did for long QT syndrome, collaboration between clinicians, molecular biologists, and biophysicists has, for CPVT as well, opened new and important areas of research that through the advancement of basic science will eventually develop new hope for the cure of human diseases.

\section{Acknowledgments}

We wish to acknowledge the helpful discussion and the constructive insights provided by Anthony F. Lai (Cardiff University, Cardiff, United Kingdom) during the preparation of this article.

Address correspondence to: Silvia G. Priori, Molecular Cardiology, Maugeri Foundation, University of Pavia, Via Ferrata 8, 27100 Pavia, Italy. Phone: 39-0382-592051; Fax: 39-0382592059; E-mail: spriori@fsm.it.
1. Gillard, E.F., et al. 1992. Polymorphisms and deduced amino acid substitutions in the coding sequence of the ryanodine receptor (RYR1) gene in individuals with malignant hyperthermia. Genomics. 13:1247-1254.

2. Priori, S.G., et al. 2001. Mutations in the cardiac ryanodine receptor gene (hRyR2) underlie catecholaminergic polymorphic ventricular tachycardia. Circulation. 103:196-200.

3. Tunwell, R.E., et al. 1996. The human cardiac muscle ryanodine receptor-calcium release channel: identification, primary structure and topological analysis. Biochem. J. 318:477-487.

4. Otsu, K., et al. 1993. Chromosome mapping of five human cardiac and skeletal muscle sarcoplasmic reticulum protein genes. Genomics. 17:507-509.

5. Tiso, N., et al. 2001. Identification of mutations in the cardiac ryanodine receptor gene in families affected with arrhythmogenic right ventricular cardiomyopathy type 2 (ARVD2). Hum. Mol. Genet. 10:189-194.

6. Du, G.G., Sandhu, B., Khanna, V.K., Guo, X.H., and MacLennan, D.H. 2002. Topology of the $\mathrm{Ca} 2+$ release channel of skeletal muscle sarcoplasmic reticulum (RyR1). Proc. Natl. Acad. Sci. U. S. A 99:16725-16730.
7. Ma, J., Hayek, S.M., and Bhat, M.B. 2004. Membrane topology and membrane retention of the ryanodine receptor calcium release channel. Cell Biochem. Biophys. 40:207-224.

8. Stewart, R., Zissimopoulos, S., and Lai, F.A. 2003. Oligomerization of the cardiac ryanodine receptor C-terminal tail. Biochem. J. 376:795-799.

9. Fabiato, A., and Fabiato, F. 1978. Calcium-induced release of calcium from the sarcoplasmic reticulum of skinned cells from adult human, dog, cat, rabbit, rat, and frog hearts and from fetal and new-born rat ventricles. Ann. N. Y. Acad. Sci. 307:491-522.

10. Carl, S.L., et al. 1995. Immunolocalization of sarcolemmal dihydropyridine receptor and sarcoplasmic reticular triadin and ryanodine receptor in rabbit ventricle and atrium. J. Cell Biol. 129:672-682.

11. Wang, S.Q., Song, L.S., Lakatta, E.G., and Cheng, H. 2001. Ca2+ signalling between single L-type $\mathrm{Ca} 2+$ channels and ryanodine receptors in heart cells. Nature. 410:592-596.

12. Franzini-Armstrong, C., Protasi, F., and Ramesh, V. 1999. Shape, size, and distribution of $\mathrm{Ca}(2+)$ release units and couplons in skeletal and cardiac muscles. Biophys. J. 77:1528-1539.

13. Bers, D.M. 2004. Macromolecular complexes regulating cardiac ryanodine receptor function. J. Mol.
Cell. Cardiol. 37:417-429.

14. Marx, S.O., et al. 2001. Coupled gating between cardiac calcium release channels (ryanodine receptors). Circ. Res. 88:1151-1158.

15. Meissner, G. 2002. Regulation of mammalian ryanodine receptors. Front. Biosci. 7:d2072-d2080.

16. Masumiya, H., Wang, R., Zhang, J., Xiao, B., and Chen, S.R. 2003. Localization of the 12.6-kDa FK506-binding protein (FKBP12.6) binding site to the NH2-terminal domain of the cardiac $\mathrm{Ca} 2+$ release channel (ryanodine receptor). J. Biol. Chem. 278:3786-3792.

17. Zissimopoulos, S., and Lai, F.A. 2002. Evidence for a FKBP12.6 binding site at the C-terminus of the cardiac ryanodine receptor [abstract]. Biophys. $J$. 82:59.

18. Marx, S.O., et al. 2000. PKA phosphorylation dissociates FKBP12.6 from the calcium release channel (ryanodine receptor): defective regulation in failing hearts. Cell. 101:365-376.

19. Lehnart, S.E., Wehrens, X.H., and Marks, A.R. 2004. Calstabin deficiency, ryanodine receptors, and sudden cardiac death. Biochem. Biophys. Res. Commun. 322:1267-1279.

20. Xiao, B., et al. 2005. Characterization of a novel PKA phosphorylation site, serine-2030, reveals no 
PKA hyperphosphorylation of the cardiac ryanodine receptor in canine heart failure. Circ. Res. 96:847-855

21. Loke, J., and MacLennan, D.H. 1998. Malignant hyperthermia and central core disease: disorders of Ca2+ release channels. Am. J. Med. 104:470-486.

22. Jurkat-Rott, K., McCarthy, T., and Lehmann-Horn, F. 2000. Genetics and pathogenesis of malignant hyperthermia. Muscle Nerve. 23:4-17.

23. Jungbluth, H., et al. 2002. Autosomal recessive inheritance of RYR1 mutations in a congenital myopathy with cores. Neurology. 59:284-287.

24. Frank, J.P., Harati, Y., Butler, I.J., Nelson, T.E., and Scott, C.I. 1980. Central core disease and malignant hyperthermia syndrome. Ann. Neurol. 7:11-17.

25. Davis, M.R., et al. 2003. Principal mutation hotspot for central core disease and related myopathies in the C-terminal transmembrane region of the RYR1 gene. Neuromuscul. Disord. 13:151-157.

26. Monnier, N., et al. 2003. A homozygous splicing mutation causing a depletion of skeletal muscle RYR1 is associated with multi-minicore disease congenital myopathy with ophthalmoplegia. Hum. Mol. Genet. 12:1171-1178.

27. Richter, M., Schleithoff, L., Deufel, T., LehmannHorn, F., and Herrmann-Frank, A. 1997. Functional characterization of a distinct ryanodine receptor mutation in human malignant hyperthermia-susceptible muscle. J. Biol. Chem. 272:5256-5260.

28. Yang, T., Ta, T.A., Pessah, I.N., and Allen, P.D. 2003. Functional defects in six ryanodine receptor isoform-1 (RyR1) mutations associated with malignant hyperthermia and their impact on skeletal excitation-contraction coupling. J. Biol. Chem. 278:25722-25730.

29. Wehner, M., Rueffert, H., Koenig, F., and Olthoff, D. 2004. Functional characterization of malignant hyperthermia-associated RyR1 mutations in exon 44 , using the human myotube model. Neuromuscul. Disord. 14:429-437.

30. Tilgen, N., et al. 2001. Identification of four novel mutations in the $\mathrm{C}$-terminal membrane spanning domain of the ryanodine receptor 1: association with central core disease and alteration of calcium homeostasis. Hum. Mol. Genet. 10:2879-2887.

31. Avila, G., and Dirksen, R.T. 2001. Functional effects of central core disease mutations in the cytoplas- mic region of the skeletal muscle ryanodine receptor. J. Gen. Physiol. 118:277-290.

32. Avila, G., O'Brien, J.J., and Dirksen, R.T. 2001. Excitation-contraction uncoupling by a human central core disease mutation in the ryanodine receptor. Proc. Natl. Acad. Sci. U. S. A. 98:4215-4220.

33. Dirksen, R.T., and Avila, G. 2002. Altered ryanodine receptor function in central core disease: leaky or uncoupled $\mathrm{Ca}(2+)$ release channels [review]? Trends Cardiovasc. Med. 12:189-197.

34. Coumel, P., Fidelle, J., Lucet, V., Attuel, P., and Bouvrain, Y. 1978. Catecholaminergic-induced severe ventricular arrhythmias with Adams-Stokes syndrome in children: report of four cases. Br. Heart J. 40:28-37.

35. Leenhardt, A., et al. 1995. Catecholaminergic polymorphic ventricular tachycardia in children. A 7-year follow-up of 21 patients. Circulation. 91:1512-1519.

36. Priori, S.G., et al. 2002. Clinical and molecular characterization of patients with catecholaminergic polymorphic ventricular tachycardia. Circulation. 106:69-74.

37. Swan, H., et al. 1999. Arrhythmic disorder mapped to chromosome $1 \mathrm{q} 42-\mathrm{q} 43$ causes malignant polymorphic ventricular tachycardia in structurally normal hearts. J. Am. Coll. Cardiol. 34:2035-2042.

38. Lahat, H., et al. 2001. Autosomal recessive catecholamine- or exercise-induced polymorphic ventricular tachycardia. Circulation. 103:2822-2827.

39. Lahat, H., et al. 2001. A missense mutation in a highly conserved region of CASQ2 is associated with autosomal recessive catecholamine-induced polymorphic ventricular tachycardia in Bedouin families from Israel. Am. J. Hum. Genet. 69:1378-1384.

40. Postma, A.V., et al. 2002. Absence of calsequestrin 2 causes severe forms of catecholaminergic polymorphic ventricular tachycardia. Circ. Res. 91:e21-e26.

41. Cerrone, M., et al. 2004. Clinical and molecular characterization of a large cohort of patients affected with catecholaminergic polymorphic ventricular tachycardia [abstract]. Circulation. 110(Suppl. 3):552.

42. Priori, S.G., et al. 2004. Association of long QT syndrome loci and cardiac events among patients treated with beta-blockers. JAMA. 292:1341-1344.

43. Priori, S.G., et al. 2003. Risk stratification in the long-QT syndrome. N. Engl. J. Med. 348:1866-1874. 44. Choi, G., et al. 2004. Spectrum and frequency of cardiac channel defects in swimming-triggered arrhythmia syndromes. Circulation. 110:2119-2124.

45. Tester, D.J., Spoon, D.B., Valdivia, H.H., Makielski, J.C., and Ackerman, M.J. 2004. Targeted mutational analysis of the RyR2-encoded cardiac ryanodine receptor in sudden unexplained death: a molecular autopsy of 49 medical examiner/coroner's cases. Mayo Clin. Proc. 79:1380-1384

46. Jiang, D., Xiao, B., Zhang, L., and Chen, S.R. 2002. Enhanced basal activity of a cardiac $\mathrm{Ca} 2+$ release channel (ryanodine receptor) mutant associated with ventricular tachycardia and sudden death. Circ. Res. 91:218-225.

47. Jiang, D., et al. 2004. RyR2 mutations linked to ventricular tachycardia and sudden death reduce the threshold for store-overload-induced $\mathrm{Ca} 2+$ release (SOICR). Proc. Natl. Acad. Sci. U. S. A. 101:13062-13067.

48. Wehrens, X.H., et al. 2003. FKBP12.6 deficiency and defective calcium release channel (ryanodine receptor) function linked to exercise-induced sudden cardiac death. Cell. 113:829-840

49. George, C.H., Higgs, G.V., and Lai, F.A. 2003 Ryanodine receptor mutations associated with stress-induced ventricular tachycardia mediate increased calcium release in stimulated cardiomyocytes. Circ. Res. 93:531-540.

50. Lowri, T.N., George, C.H., and Anthony, L.F. 2004 Functional heterogeneity of ryanodine receptor mutations associated with sudden cardiac death. Cardiovasc. Res. 64:52-60.

51. Gomez, A.M., and Richard, S. 2004. Mutant cardiac ryanodine receptors and ventricular arrhythmias: is 'gain-of-function' obligatory? Cardiovasc. Res. 64:3-5.

52. Kieval, R.S., Butler, V.P., Jr., Derguini, F., Bruening, R.C., and Rosen, M.R. 1988. Cellular electrophysiologic effects of vertebrate digitalis-like substances. J. Am. Coll. Cardiol. 11:637-643.

53. Priori, S.G., and Corr, P.B. 1990. Mechanisms underlying early and delayed afterdepolarizations induced by catecholamines. Am. J. Physiol. 258:H1796-H1805

54. Cerrone, M., et al. 2005. Bidirectional ventricular tachycardia and fibrillation elicited in a knock-in mouse model carrier of a mutation in the cardiac ryanodine receptor (RyR2). Circ. Res. 96:e77-e82. 